\title{
Investigation of Self-Assembled Monolayer by Atom Probe Microscopy
}

\author{
B.Gault, * W. Yang, * K.R. Ratinac, * R.Zheng, * F. Braet, * and S.P. Ringer* \\ * Australian Key Centre for Microscopy and Microanalysis, Madsen Building F09, The University of \\ Sydney, NSW 2006, Australia.
}

The molecular-level control over surface modification afforded by alkanethiol self-assembled monolayers (SAMs) makes them an attractive form of nanotechnology for so called 'bottom up' nanofabrication [1]. Improve and controlling the design of structures at such a scale requires highly accurate characterisation methods and techniques. X-ray photoelectron spectroscopy (XPS) is quantitative and sensitive to elemental and functional group composition is commonly used for surface analysis, but suffers from a chemical resolution often unable to determine the overall chemical structures of materials adsorbed on surfaces. Mass spectrometry is ideal for providing chemical structure [2], but its limited spatial resolution does not permit to resolve clearly SAM interfaces.

Atom Probe Microscopy (APM) is an analytical microscopy technique that provides the 3D distribution of atoms with a sub-nanometre resolution, utilised to characterise materials on an ultrafine scale [3]. Combining both outstanding chemical sensitivity and atomic spatial resolution, APT seems uniquely positioned to provide 3-D imaging and quantitative compositional information on SAMs, as demonstrated by the exploratory work of Prosa et al. on various organic materials analysed in atom probe.

In APT, single atoms of the apex of a needle shape specimen are successively ionised and projected from the surface of a specimen (Fig.1 (a)), due exclusively to the application of a very intense electric field, of the order of $10^{10} \mathrm{Vm}-1$. A simple inverse point-projection algorithm is then used to reconstruct the analysed volume in three dimensions in the real-space (Fig.1(b)). Three projection parameters are involved in the reconstruction. We have recently proposed a method to determine these parameters in order to get a very accurate reconstruction [5]. During this presentation we will show how this methodology is used to calibrate of the reconstruction of our preliminary investigation of alkanethiol SAM formed on pure Ni tips.

[1] J.C. Love et al., Chem. Rev. 105, 1103 (2005).

[2] W. Yang et al., Anal. Chem. 75, 6741 (2003).

[3] T.F. Kelly, M.K. Miller, Rev. Sci. Instr. 78, (2007) 031101.

[4] T. J. Prosa et al., Microsc. Microanal. 13, (2007), 190.

[5] B. Gault et al., Microsc. Microanal. 14, (2008) 296-305. 


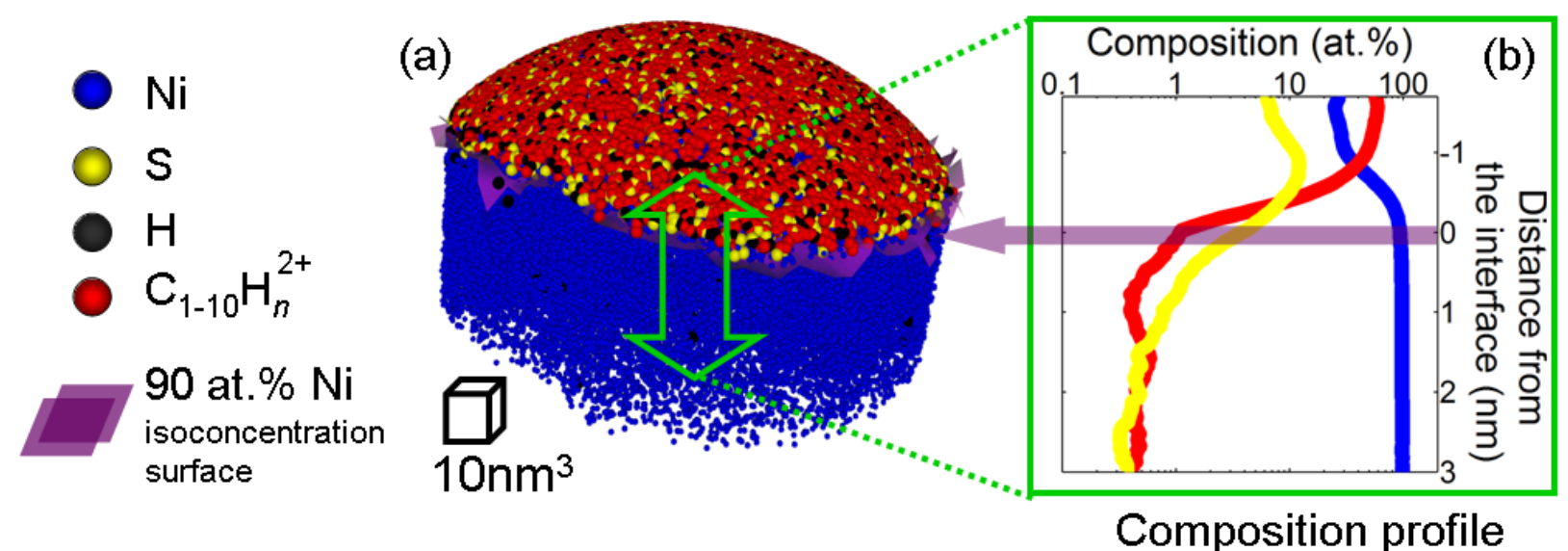

Figure 1. (a) 3D tomographic atom map of a decanethiol SAM on $\{001\} \mathrm{Ni}$. The interface, defined as a $90 \% \mathrm{Ni}$ isoconcentration surface (purple), is shown. (b) Composition-depth profile where the zero point is the interface defined in (a); for clarity, the composition in $\mathrm{H}$ was not shown in here.

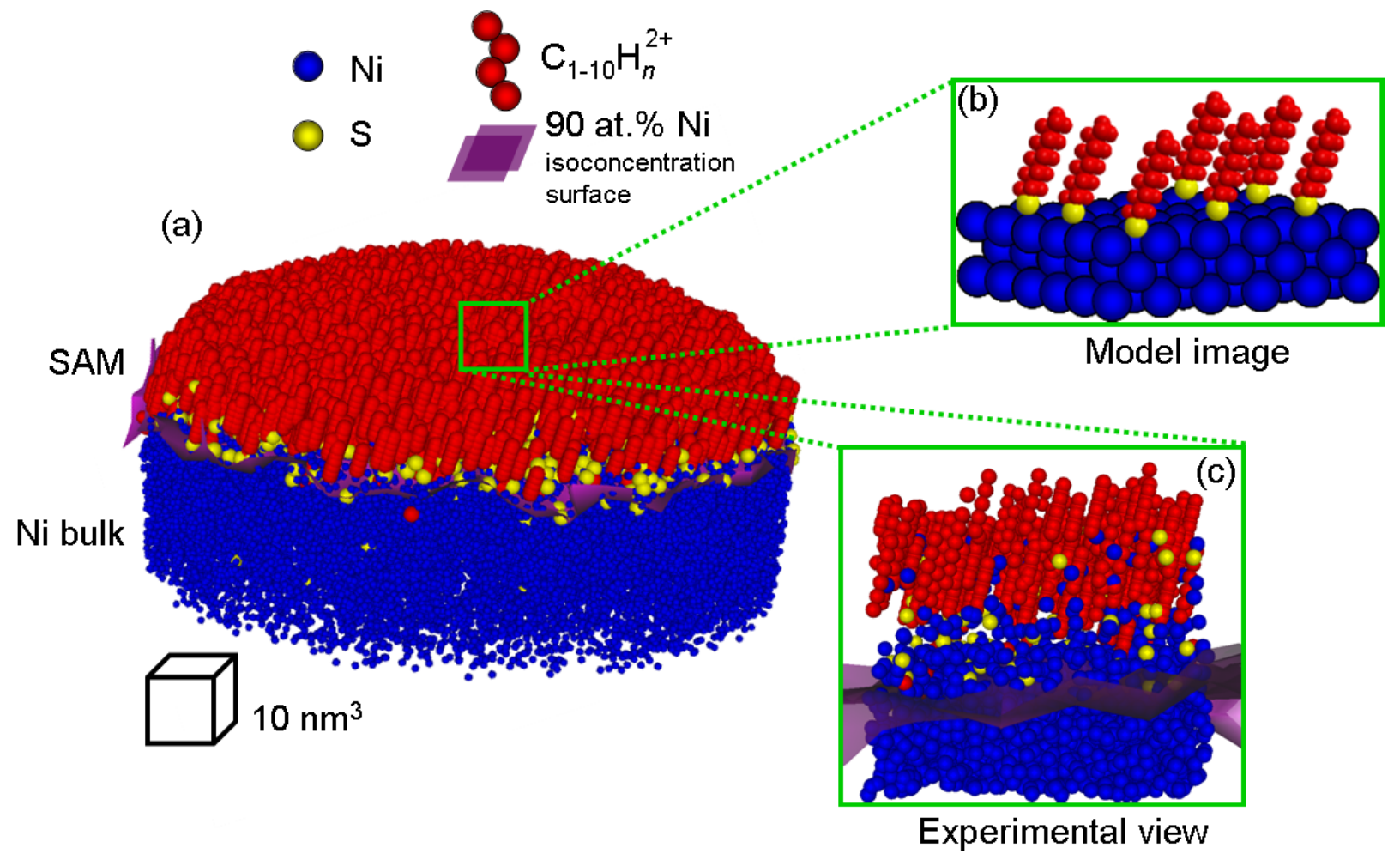

Fig.2: (a) Same 3D tomographic atom map of a decanethiol SAM on $\{001\}$ Ni as in Figure 1 (a) after the rendering process. (b) Model decanethiol SAM $/\{001\}$ Ni surface and (c) high-resolution atom map resolving individual molecules. 